\title{
NOTE E RECENSIONI
}

\author{
Kate Pickett e Richard G. Wilkinson, La misura dell'anima. Perché le \\ disuguaglianze rendono le società più infelici, Feltrinelli, Milano, 2009 \\ pagine $304^{*}$
}

Secondo i due autori di questo libro che, oltre ad essere degli studiosi sociali, sono specializzati anche in epidemiologia, alla base dei più diffusi malesseri sociali si trova la disuguaglianza: infatti, se nei vari paesi del mondo si è in presenza di mali come la violenza, l'ignoranza, il disagio psichico, orari di lavoro eccessivamente lunghi e penosi, così come si riscontra un elevato numero di malati, di detenuti, di tossicodipendenti, di ragazze madri e, perfino, di obesi, all'origine di tutto ciò che - come è facile immaginare - comporta un elevato tasso di infelicità, sembra esserci un altrettanto elevato divario tra le classi ricche e quelle povere.

Tale affermazione è dimostrata in questo libro sulla base di una serie molto ampia di dati che sono stati elaborati dagli autori nel corso di trent'anni di ricerche, indagini e comparazioni tra informazioni statistiche relative ai principali paesi, sviluppati e non. I risultati delle loro elaborazioni econometriche, inoltre, sono suffragati da un'amplissima bibliografia, costituita da quasi 400 fra libri, articoli e altre pubblicazioni scientifiche, appartenenti a discipline diverse che vanno dall'economia alla medicina.

Da una simile analisi, condotta ed esposta in modo estremamente efficace, emerge un'immagine del mondo in cui viviamo estremamente diversa da quella che si è solitamente portati a immaginare. Infatti, si tende a ritenere che la crescita economica, intesa appunto come un mero aumento del prodotto ottenuto dai vari sistemi economici e del reddito delle comunità che risiedono in essi, produca in modo automatico l'effetto di rendere le nazioni più soddisfatte e più felici. Ed invece gli autori, inserendosi nel recente dibattito sulla significatività di taluni indicatori di contabilità nazionale (quali il Prodotto interno lordo), sostengono che non è assolutamente così, anche perché i malesseri che sarebbero generati dalla disuguaglianza socio-economica, tendono a coinvolgere tutti i ceti sociali: non solo quelli più svantaggiati, ma anche quelli che si collocano al vertice della scala sociale.

La soluzione prospettata da Pickett e Wilkinson nell'ultimo capitolo del libro è altrettanto chiara: se s'intende avviare un nuovo ciclo di sviluppo socioeconomico, magari cogliendo l'occasione offerta dalla grave crisi economicofinanziaria che si sta attraversando a livello mondiale, per cambiare obiettivo

\footnotetext{
* Traduzione italiana di The Spirit Level. Why More Equal Societies Almost Always Do Better, Penguin, London, 2009.
} 
di politica economica perseguendo il reale benessere e la qualità della vita occorre che i vari soggetti responsabili, tra cui anche le grandi imprese multinazionali e i sindacati, si rendano conto che è necessario intervenire urgentemente e con determinazione, al fine di ridurre la forbice sociale che è cresciuta a dismisura negli ultimi decenni, sia al di qua che al di la dell'Atlantico. Gli interventi da adottare dovrebbero pertanto puntare a redistribuire sia il reddito che le opportunità, prendendo ispirazione da paesi come il Giappone e quelli scandinavi, che sono quelli in cui la disuguaglianza è minore.

L'Italia, invece, si colloca ai primi posti della graduatoria degli oltre venti paesi per i quali sono risultati disponibili i dati su cui è stato calcolato il livello di sperequazione dei redditi. Questo significa evidentemente che le misure da adottare dovrebbero essere impiegate in misura particolarmente intensa e prolungata affinché possano ottenere il risultato sperato, anche perché nel nostro paese alla disuguaglianza sociale si affianca una netta disuguaglianza territoriale, riconducibile all'annoso e tuttora esistente dualismo fra Centro-Nord e Sud.

Il riferimento ad una politica di riequilibrio territoriale sembra del resto confermare gli assunti del libro, giacché anche la teoria economica convenzionale sostiene da tempo che quando le disparità territoriali assumono dimensioni particolarmente accentuate - come nel caso italiano - esse causano gravi problemi all'intero sistema economico, rendendo ancora più difficili da raggiungere i tradizionali obiettivi della politica macroeconomica, quali la piena occupazione, il contenimento del tasso d'inflazione, nonché l'equilibrio del bilancio pubblico e quello della bilancia dei pagamenti.

Da qui la personale indicazione circa la convenienza economico-finanziaria per il progetto di federalismo fiscale che si sta per realizzare, di perseguire anche l'obiettivo della riduzione della disuguaglianza sul piano territoriale secondo schemi nuovi e più coerenti.

Aurelio Bruzzo

\section{Fabio Berton, Matteo Richiardi e Stefano Sacchi, Flex-insecurity. Perché in Italia la flessibilità diventa precarietà, il Mulino, Bologna, 2009 pagine 360}

Le società moderne sono caratterizzate da un elevato tasso di complessità che sempre più mette a dura prova i processi di regolazione deputati a mantenere parvenze di equilibrio. L'infittirsi dei rapporti e degli scambi, la perdita di senso delle distanze e l'azzeramento dei lag temporali, l'accesso immediato all'informazione, la molteplicità dei ruoli che ciascuno è chiamato a rivestire generano inevitabilmente attriti e conflitti che non sempre è facile ridurre e comporre.

Se il cittadino consumatore ha potuto trarre vantaggio dai processi di riorganizzazione produttiva, di evoluzione tecnologica, di internazionalizzazione degli scambi e di allargamento della concorrenza godendo della smisurata varietà dei prodotti a disposizione, del loro ridotto costo e dall'incrementata ac- 
cessibilità, tali mutamenti non hanno sortito effetti positivi rispetto al cittadino lavoratore, prioritariamente quando residente nei Paesi ricchi.

La riduzione della sicurezza occupazionale è l'effetto che tutti possono, se non - fortunatamente - sperimentare direttamente, percepire come più tangibile di un tempo. La crescente necessità di flessibilità prestazionale richiesta dai sistemi produttivi, declinata in tutte le accezioni possibili, si trasforma per molti in precarietà occupazionale $\mathrm{e}$, spesso, esistenziale.

La nuova strutturazione del mercato mondiale è compatibile con il modello di sicurezza sociale europeo o il futuro che ci attende sarà, senza possibilità di alternative, quello di una società del rischio più individualista e americanizzata?

In alcuni Paesi del nord Europa il binomio flessibilità/precarietà è stato scisso per teorizzarne la proficua coniugazione nella flexicurity, la quale vuole tenere insieme la flessibilità con la sicurezza sociale e dell'occupazione. Il titolo del libro di Berton, Richiardi e Sacchi, con la torsione applicata al termine, contestualizza la situazione italiana. È questo sicuramente un ottimo e prezioso volume che si rivolge ad un pubblico quanto mai vario, come gli autori stessi dichiarano nella presentazione, offrendo elementi reali di conoscenza riguardo ad un argomento che ormai da anni anima il dibattito tra gli esperti, ma anche dei talk show di prima serata televisiva e che, con la crisi economica imperante, si è ancor più rivitalizzato.

L'articolazione del volume consente al lettore interessato e curioso di prendere consapevolezza dell'argomento in tutte le sue sfaccettature come del resto permette agli specialisti di guardare oltre il proprio orticello referenziale: il giuslavorista rileverà una documentata base empirica che illustra come le norme trasformino la realtà (e che consentirebbe esercizi di valutazione tanto lontani dalla pratica normativa nazionale); l'economista del lavoro, piuttosto che lo statistico, troveranno documentati i passaggi normativi, assolutamente non neutrali, che determinano i mutamenti e le persistenze; l'appendice sinottica su contratti e ammortizzatori sociali sarà di estrema utilità ai giornalisti che non avranno tempo per affrontare le oltre trecento, godibili, pagine del libro.

Gli autori sostengono la tesi, del tutto condivisibile, di come precarietà e flessibilità non debbano per forza essere sinonimi, ma anche di come questa eventualità diventi una realtà per un numero non trascurabile di lavoratori quando le regole del "gioco" sono quelle che vigono nel nostro Paese. I cambiamenti apportati nel corso del tempo alle regole del mercato del lavoro, per adeguare le modalità delle prestazioni all'evolversi delle necessità del mondo della produzione e dei servizi, non sono state adeguatamente supportate da conformi modifiche del sistema di welfare, che già di suo non aveva caratteristiche di universalità e di inclusività, anzi. I lavoratori atipici, cioè i dipendenti non impiegati con contratti a tempo pieno e indeterminato e i parasubordinati, soffrono in misura maggiore (ma non esclusiva dato che non tutti gli atipici sono precari e non tutti i precari sono atipici) di una discontinuità delle carrie- 
re, di uno svantaggio retributivo e previdenziale, di una difficoltà di accesso al sistema delle tutele che ne rendono effettivamente precaria l'esistenza.

La sequenza dei capitoli costituisce una guida all'analisi del problema: dalla necessaria azione definitoria a livello concettuale dei termini essenziali implicati nella discussione si passa alla propedeutica trattazione dell'evoluzione normativa che ha interessato l'ambito delle politiche del lavoro; segue poi l'analisi empirica, condotta sui dati del Work Histories Italian Panel, che tende a sostanziare quantitativamente le dimensioni lungo le quali viene analizzata la precarietà e cioè l'analisi delle carriere lavorative (discontinuità lavorativa e contrattuale), le retribuzioni e la contribuzione, il sistema delle tutele e la loro concreta fruibilità da parte dei lavoratori; due capitoli di approfondimento, con simulazioni ed ulteriori analisi empiriche, sono poi dedicati al temi del salario differito (la pensione) e della soddisfazione (vi sono dei vantaggi che ripagano dei rischi?) dei lavoratori atipici.

Il lavoro dei ricercatori, che fanno riferimento al LABORatorio Revelli di Torino, non si ferma all'individuazione dei sintomi e dei segni patognomonici ed alla loro misurazione, azione che li mette in grado di formulare una diagnosi precisa e numericamente circostanziata, ma li spinge anche a ipotizzare gli interventi che potrebbero in qualche misura fungere da cura $\mathrm{o}$, in altri termini, a portare a superare la flex-insicurity e a transitare nella flexicurity.

Le proposte di intervento (non una, ma tre diversamente modulate) sono dettagliate fino alla stima della previsione di spesa che ciascuna di loro comporterebbe se adottata. I cardini attorno ai quali però tutte ruotano sono essenzialmente due: la rimozione dei vantaggi che inducono le imprese ad utilizzare i contratti atipici in funzione di una riduzione del costo del lavoro (risultato ottenibile attraverso l'imposizione di aliquote contributive uniche, l'introduzione di un salario minimo e di un'indennità di terminazione o fine rapporto); una riforma del sistema di protezione sociale che si richiami ad un'impostazione universalistica e automatica, "che comporti dunque il riconoscimento di effettivi diritti soggettivi in capo a tutti i partecipanti al mercato del lavoro".

Tutte e tre le proposte tendono ad affermare il "principio per cui il mantenimento del reddito in caso di perdita o assenza di lavoro è un diritto per tutti i lavoratori', l'ultima di esse è quella che più radicalmente ristruttura la rete di protezione sociale giungendo sino alla proposta dell'istituzione del reddito minimo garantito quale strumento ultimo di lotta alla povertà.

Le cornici che delineano le ipotesi di riforma, come del resto le analisi attorno ai nodi problematici del sistema esistente, sono del tutto condivisibili mentre invitano ad una più approfondita discussione le fini tecnicalità applicative necessarie a rendere operativi gli interventi proposti.

$\grave{E}$ un libro che alcuni leggeranno per puro piacere e che molti sarebbero tenuti a studiare come dovere.

Maurizio Rasera 